\title{
miR-519d-mediated downregulation of STAT3 suppresses breast cancer progression
}

\author{
XIN DENG, YI ZHAO and BAOSHENG WANG \\ Department of Pancreatic and Breast Surgery, Shengjing Hospital of China Medical University, \\ Shenyang, Liaoning, P.R. China
}

Received March 19, 2015; Accepted June 5, 2015

DOI: $10.3892 /$ or.2015.4160

\begin{abstract}
MicroRNAs (miRNAs) play important roles in the the gene regulation of carcinogenesis including breast cancer. miR-519d has been studied in various types of cancer, but its role in breast cancer remains unclear. In the present study, we investigated the expression and biological function of $\mathrm{miR}$ $519 d$ in breast cancer. Using quantitative RT-PCR (RT-qPCR) analysis, we analyzed the expression of miR-519d in breast cancer tissues and cell lines. It was shown that miR-519d expression was decreased in cancer tissues and cell lines compared with their controls. Overexpression of miR-519d inhibited cell proliferation and invasion, and induced apoptosis of breast cancer cells. Signal transducer and activator of transcription 3 (STAT3) was predicted as a target gene of miR-519d and it was verified by the luciferase reporter assay. Additionally, STAT3 mRNA and protein expression levels were downregulated in the cells with miR-519d overexpression as determined by RT-qPCR and western blotting. Taken together, the results indicated that miR-519d functions as a tumor suppressor in breast cancer by suppressing STAT3 expression.
\end{abstract}

\section{Introduction}

MicroRNAs (miRNAs), a type of non-coding RNAs, play important regulatory roles in gene expression by binding to the 3'-untranslated region (3'UTR) of target mRNAs and lead to mRNA degradation or translation inhibition (1). miRNAs are aberrantly expressed in different types of tumors and play act as tumor suppressors or oncogenes depending on their target genes (2-4). The roles of miRNAs in tumor include cell growth, death, autophagy, apoptosis, angiogenesis and differentiation, metastasis and therapy resistance in tumor progression (5-7).

Breast cancer is one of the leading causes of cancer mortality among women worldwide (8). A number of miRNAs

Correspondence to: Dr Xin Deng, Department of Pancreatic and Breast Surgery, Shengjing Hospital of China Medical University, 36 Heping Street, Shenyang, Liaoning 110004, P.R. China E-mail: dengxin14@yeah.net

Key words: miR-519d, STAT3, breast cancer, metastasis, apoptosis appear to be involved in breast cancer progression (5-7). miR$519 \mathrm{~d}$ is reported as a negative regulator of tumor progression in chondrosarcoma (9), osteosarcoma (10), ovarian cancer (11) and hepatocellular carcinoma $(12,13)$. However, its expression and cell function in breast cancer remains to be determined.

Signal transducer and activator of transcription 3 (STAT3) is a key cytoplasmic transcription factor that is usually activated by cytokine receptors and tyrosine kinase in the cells (14). STAT3 plays an important role in early embryogenesis and the regulation of various types of cells (15-16). The role of STAT3 in breast cancer has been widely studied and aberrant activation of STAT3 can also promote breast cancer formation and progression (15-16). STAT3 activation is frequently observed in primary breast cancers and is associated with poor prognosis and invasiveness (15-16). It has been shown that miRNAs regulate STAT3 expression in cancer cells (17), but its regulation by miRNAs needs to be further elucidated in breast cancer.

In the present study, we examined the potential role of miR-519d in breast cancer. The miRNA prediction software indicated that STAT3 may be the downstream target of miR-519d. Furthermore, the correlation between the expression of miR-519d and STAT3 in breast cancer samples was explored.

\section{Materials and methods}

Patients and tumor tissues. Human breast cancer tissues and matched normal adjacent breast specimens from the patients diagnosed with breast cancer were obtained at the Shengjing Hospital of China Medical University between 2009 and 2013. The diagnosis was based on pathological evidence and the specimens were immediately snap-frozen and stored at $-80^{\circ} \mathrm{C}$. None of the patients received chemotherapy or radiotherapy before the surgical excision. All the patients provided written informed consent for the use of their tissues. The study protocol was approved by the Ethics Committee of Shengjing Hospital.

Cell lines and culture. Human MCF7, BT474, SKBR3 and MDA231 breast cancer cell lines were primarily obtained from the American Type Culture Collection (ATCC; Manassas, VA, USA) and were maintained in the medium according to the instructions from ATCC. MCF10A cells were cultured in Dulbecco's modified Eagle's medium (DMEM)/F12 with $10 \%$ fetal bovine serum (FBS) and antibiotics (100 U/ml penicillin and $100 \mu \mathrm{g} / \mathrm{ml}$ streptomycin sulfate, EGF and insulin). The 
cells were grown in a humidified incubator at $37^{\circ} \mathrm{C}$ with $5 \% \mathrm{CO}_{2}$.

Quantitative RT-PCR. Total RNA was isolated from breast cancer tissues or cells using TRIzol (Invitrogen-Life Technologies, Carlsbad, CA, USA). miR-519d and U6 were polyadenylated using a poly-A polymerase based First-Strand Synthesis kit (Takara Bio, Japan) following the manufacturer's instructions. To quantify the STAT3 and GAPDH mRNA levels, $1 \mu \mathrm{g}$ of total RNA was subjected to First-Strand cDNA synthesis using a PrimeScript RT Reagent kit (Takara). The qPCR was performed using SYBR-Green PCR master mix (Takara) on the ABI 7500HT system. U6 or GAPDH were used as an endogenous control. All the primers were ordered from Invitrogen. The relative fold expression was calculated with the $2^{-\Delta \Delta C T}$ method. The RT-qPCR reactions were run in triplicate (18).

Transfection. miR-519d, anti-miR-519d and their controls were designed and synthesized by RiboBio Co. (Guangzhou, China). The cells were transfected with miRNA using Lipofectamine 2000 (Invitrogen-Life Technologies). Briefly, breast cancer cells were seeded in 12-well plates and grown until they reached $40 \%$ confluence prior to transfection, and RNA and protein were extracted $48 \mathrm{~h}$ later. The final concentration of miR-519d mimic and anti-miR-519d was $50 \mathrm{nM}$. Lentiviral miR-519d (LV-miR-519d) and empty lentiviral vector (LV-miR-control) were constructed by Genechem Co. (Shanghai, China) and were infected into the breast cancer cells according to the manufacturer's instructions.

MTT assay. An MTT assay was employed to detect the growth of breast cancer cells and the growth curve was delineated. Briefly, $2.5 \times 10^{3}$ cells/well were seeded in a 96-well plate and allowed to adhere. At different time-points, $20 \mu \mathrm{l}$ of the MTT solution was added to each well $(5 \mathrm{mg} / \mathrm{ml}$ and $0.5 \%$ MTT) and the cells were continued to culture for $4 \mathrm{~h}$. After the incubation, the supernatant was discarded and $150 \mu \mathrm{l}$ dimethyl sulfoxide was added to each well, and the culture plate was agitated at low speed for 10 min until the crystal dissolved completely. The ELISA reader was used to measure the absorbance at $570 \mathrm{~nm}$.

Apoptosis assay. For apoptosis analysis, the cells were collected, washed twice with cold phosphate-buffered saline and resuspended in binding buffer at a cell density of $1 \times 10^{6} / \mathrm{ml}$. The cells were then stained with Annexin V-FITC and propodium iodide according to the manufacturer's instructions. The signal was obtained by a FACSCalibur flow cytometer (BD Biosciences, San Jose, CA, USA) and was analyzed with CellQuest software.

Cell cycle analysis. MCF7 or SKBR3 cells were transfected with miR-519 or miR-control and the cells were collected for cell cycle analysis. The cells were fixed in $70 \%$ ethanol overnight at $-20^{\circ} \mathrm{C}$, and treated with DNA staining solution. Cell cycle analysis was performed using flow cytometry.

Matrigel invasion assay. MCF7 or SKBR3 cells were transfected with miR-519 or miR-control and were collected for the invasion assay using a Matrigel-coated chamber (24-well plates, 8-mm pore size; Corning Inc., Corning, NY, USA). Briefly, breast cancer cells transfected with miR-519 or miR-control $\left(5 \times 10^{4}\right)$ were seeded in the upper chamber at $37^{\circ} \mathrm{C}$ with the medium with 0.1 and $20 \%$ FBS in the lower wells. After $48 \mathrm{~h}$, the chambers were removed and the non-invading cells were then wiped with cotton swabs. Invaded cells at the bottom of the membrane were stained with $0.1 \%$ crystal violet and counted under microscopic observation with five fields.

Western blot analysis. Breast cancer cells were collected after $48 \mathrm{~h}$ treatment with $50 \mathrm{nM}$ miR-519d mimic or anti-miR-519d and the controls. Protein extraction, SDS-PAGE gel electrophoresis and western blotting were performed as previously described. Several different primary antibodies were used including STAT3 (Cell Signaling Technology, Danvers, MA, USA) and GAPDH (Santa Cruz Biotechnology, Inc., Santa Cruz, CA, USA). Secondary antibody incubations were performed for $2 \mathrm{~h}$ at room temperature and the protein bands were visualized on X-ray film using an enhanced chemiluminescent ECL substrate.

Luciferase reporter assay. A total of $1 \times 10^{4}$ cells were seeded in 96-well plates in $200 \mu \mathrm{l}$ medium. A total of $100 \mathrm{ng}$ wild-type or mutant reporter constructs were co-transfected combined with $50 \mathrm{nM}$ miR-519d or miR-control using the Lipofectamine 2000 transfection reagent into the breast cancer cells according to the manufacturer's instruction. After $48 \mathrm{~h}$, luciferase activity was measured with the Dual-Luciferase reporter assay system (Promega, Madison, WI, USA). Firefly luciferase activity was then normalized to the corresponding Renilla luciferase activity.

Breast cancer xenograft model. Tumorigenicity of $1 \times 10^{7}$ cells in mammary fat pads of 4-week-old female nude mice (BALB/c-nude) was assessed. The tumor size was measured with a vernier caliper every five days and the tumor volumes were calculated using the formula: (length $\mathrm{x}$ width $\left.{ }^{2}\right) / 2 \mathrm{~mm}^{3}$. The use of nude mice complied with the guide for the use of Laboratory Animals and the study was approved by the Animal Care and Use Committee of Shengjing Hospital.

Statistical analysis. The results are presented as mean \pm standard deviation (SD). Statistical significance was determined using the Student's t-test. $\mathrm{P}<0.05$ was considered statistically significant.

\section{Results}

The expression of miR-519d is lower in breast cancer patients and cells. Firstly, we examined the expression of miR-519d in human breast cancer specimens by quantitative RT-PCR (RT-qPCR). The expression levels of miR-519d were analyzed and they were downregulated in breast cancer tissues compared with normal samples (Fig. 1A). Cancer tissues were classified as T1, T2, T3 and T4, and we found that miR-519d expression was higher in stage T1, whereas stages T2, T3 and T4 had lower levels, showing a significant correlation of miR-519d with $\mathrm{T}$ stage of breast cancer (Fig. 1B). Breast cancer tissues were classified as non-metastasis and metastasis, and the result 
A

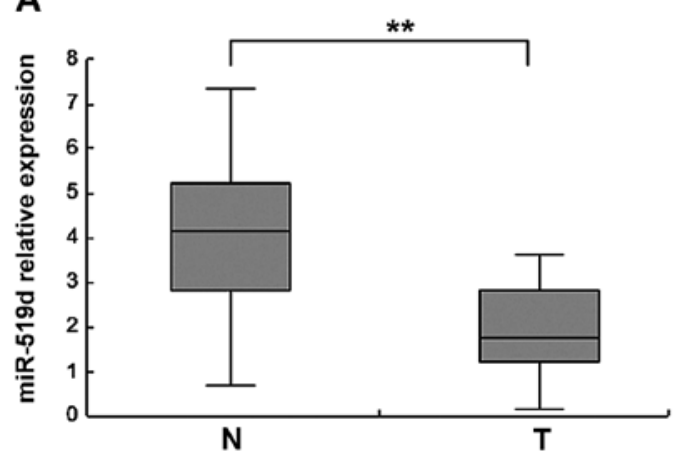

C

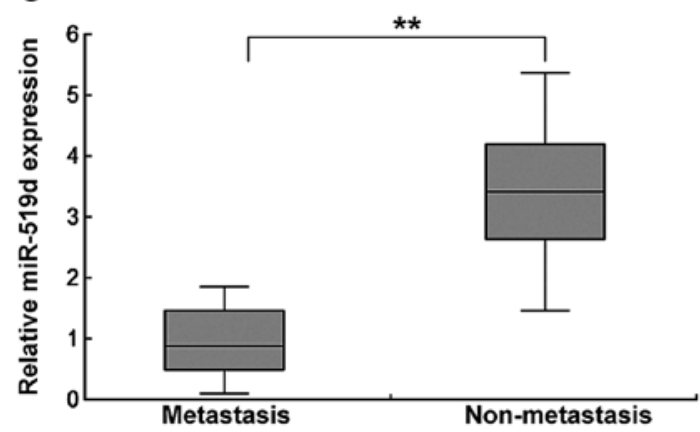

B

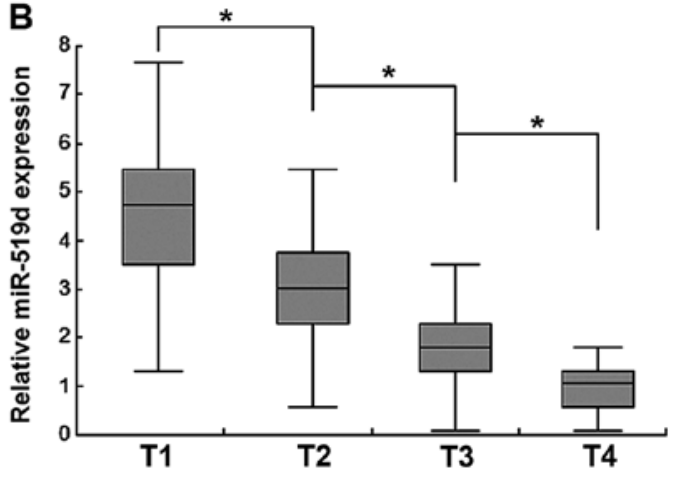

D

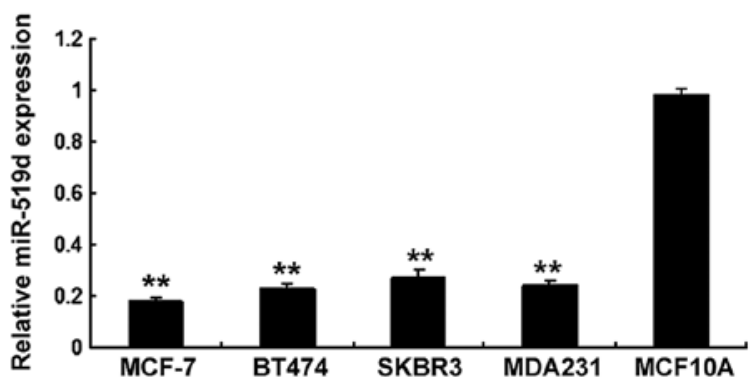

Figure 1. miR-519d expression in the human breast cancer tissues and cell lines. (A) The relative expression level of miR-519d was examined in human breast cancer tissues $(n=38)$ and matched adjacent non-cancer breast tissues $(n=38)$ by RT-qPCR. T, breast cancer tissues; N, adjacent non-cancer breast tissues. (B) The expression of miR-519d was high in T1 but low in T2-T4. (C) The expression of miR-519d was lower in the metastatic tissues as compared to the non-metastatic ones. (D) The expression level of miR-519d in MCF7, BT474, SKBR3, MDA231 and MCF10A breast cancer cell lines. Data are presented in breast cancer cell lines relative to the control. U6 was used as the control for RNA loading and miRNA abundance was normalized to that of the U6 RNA. ${ }^{* *} \mathrm{P}<0.01$ and ${ }^{*} \mathrm{P}<0.05$.

A
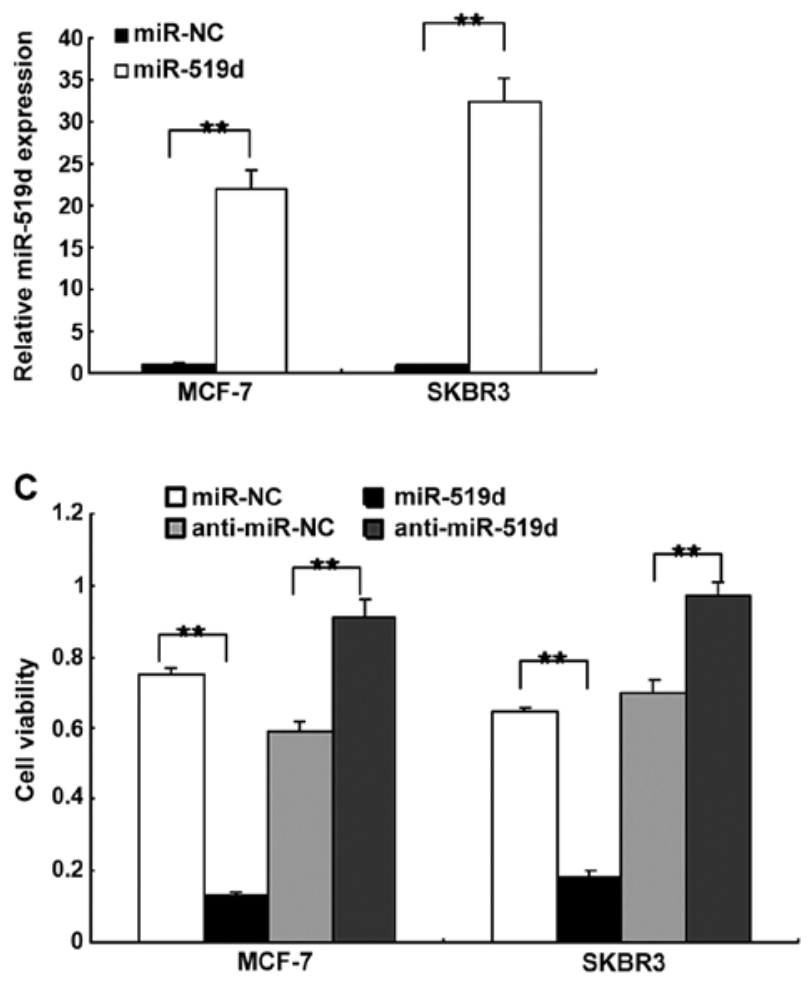

B

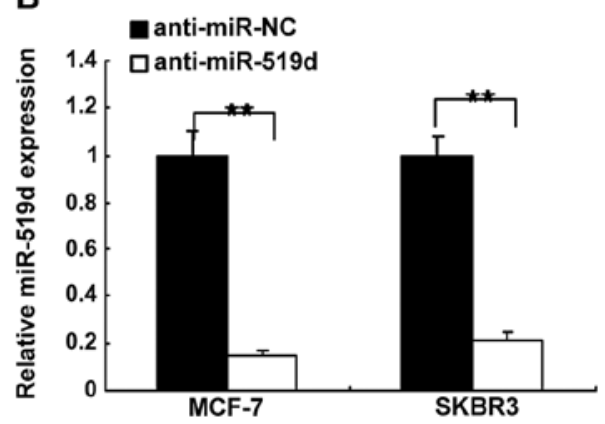

D

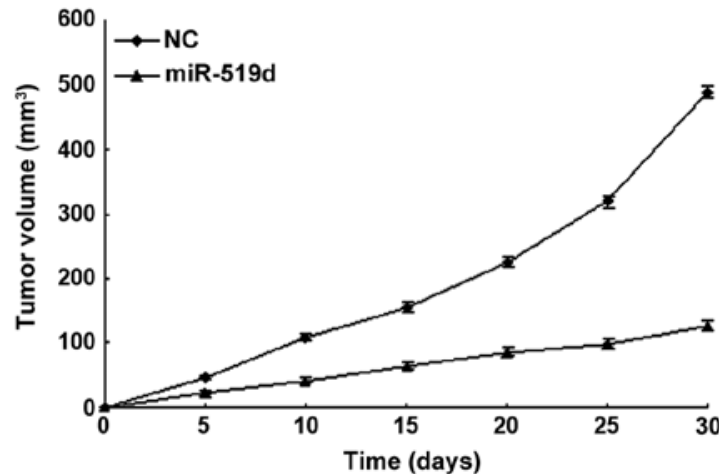

Figure 2. miR-519d inhibits the growth of breast cancer cells in vitro and in vivo. miR-519d expression level of breast cancer cells transfected with (A) miR-519d mimics (miR-519d) and (B) anti-miR-519d. Their control was examined by RT-qPCR. (C) MCF7 and SKBR3 cells were transfected with miR-519d or antimiR-519d $(50 \mathrm{nM})$ for $24 \mathrm{~h}$ and cell proliferation was examined by MTT assay. (D) miR-519d inhibited tumorigenicity in the nude mouse xenograft model. MCF7 cells were transduced with LV-miR-519d or the control. The infected MCF7 cells were then subcutaneously injected into the nude mice. The tumor growth curves were mapped. Data are represented as the mean \pm SD in 10 mice. ${ }^{* *} \mathrm{P}<0.01$ as compared to the control. 
A

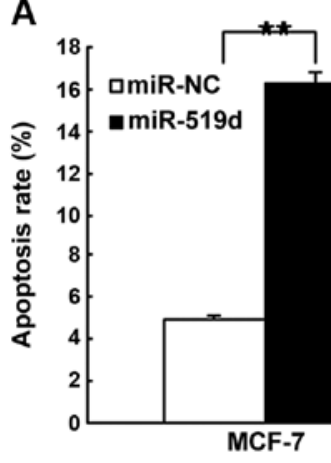

C

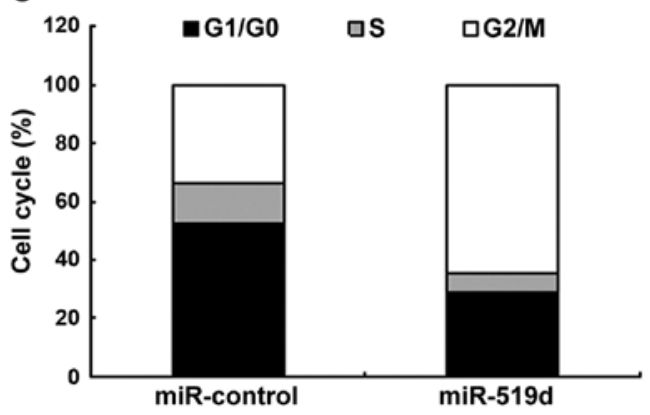

B

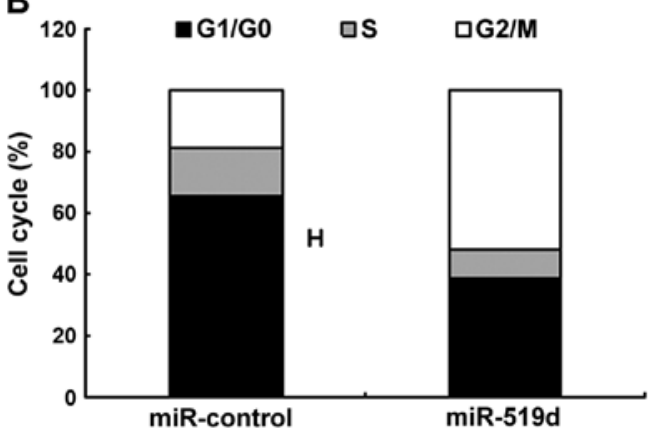

Figure 3. miR-519d induces apoptosis and cell cycle arrest in the breast cancer cells. (A) MCF7 and SKBR3 cells were transfected with miR-519d or miR-NC $(50 \mathrm{nM})$ for $48 \mathrm{~h}$ and the apoptosis was measured by Annexin V staining and flow cytometry. (B and C) The MCF7 and SKBR3 cells were treated as mentioned in (A) and the cell cycle distribution was measured by PI staining and flow cytometry. Data are presented as mean \pm SD. Results are from three independent experiments. ${ }^{* *} \mathrm{P}<0.01$ as compared to the control.

showed that miR-519d expression in the metastasis group was lower than that of the non-metastasis group (Fig. 1C). miR-519d was lower in human ovarian cancer cells compared with normal cells (Fig. 1D). These results suggested that miR$519 \mathrm{~d}$ may function as a negative regulator in the progression of human breast cancer.

miR-519d suppresses breast cancer cell proliferation. To examine the cell function of miR-519d in breast cancer cells, the MCF7 and SKBR3 cells were transfected with miR-519d mimics or the control, and the miR-519d was restored in the two cell lines (Fig. 2A). miR-519d expression was decreased in MCF7 and SKBR3 cells with anti-miR-519d (Fig. 2B). Using the MTT assay, cell proliferation was examined. The results indicated that miR-519d inhibited cell proliferation while deletion of miR-519 promoted cell proliferation in the MCF7 and SKBR3 cells (Fig. 2C). To confirm that miR-519d inhibited tumor growth, in vivo tumor models were established by implanting the MCF7 cells transfected with lentivirus-mediated miRNA-519d or negative control. Tumors with miR-519d-transfected cells grew significantly more gradually than the control (Fig. 2D). These results indicated that miR-519d suppressed breast cancer growth in vitro and in vivo.

miR-519d leads to cell apoptosis and changes cell cycle distribution in breast cancer cells. We determined whether miR-519d promoted cancer cell apoptosis or induced changes to the cell cycle distribution. MCF7 and SKBR3 cells were transfected with miR-519d mimics to observe cell apoptosis and the cell cycle using flow cytometry. It was found that the apoptotic rate increased significantly in MCF7 and SKBR3 cells compared to the control (Fig. 3A). Results from the cell cycle analysis showed that miR-519d reduced the proportion of G0/G1 and G2/M phases significantly and increased the proportion of S phase in the MCF7 and SKBR3 cells when compared with the controls (Fig. 3B and C).

miR-519d inhibits invasion of the breast cancer cells. Breast cancer cell invasiveness was assayed by a Transwell system. MCF-7 cells were transfected with the miR-519d mimics or the control, and it was found that the invasion was markedly lower in the cells with miR-519d overexpression than the cells with miR-control (Fig. 4A). Similar resutls were obtained for the SKBR3 cell line (Fig. 4B). The results showed that miR-519d inhibited breast cancer cell invasion.

miR-519d downregulates STAT3 expression in the breast cancer cells. To investigate the molecular mechanism that miR-519d regulates cell function including cell proliferation and metastasis in breast cancer cells, we used a TargetScan software that predicted the putative target genes of miR-519d. Our analysis identified that STAT3 was one of the potential targets for miR-519d (Fig. 5A). Results from the luciferase activity assay showed that the miR-519d significantly decreased the luciferase activity of with the wild-type 3'UTR, but not the mutant 3'UTR vector (Fig. 5B). miRNA regulates gene expression at the transcriptional and translational levels. However, whether miR-519d downregulated STAT3 mRNA or protein remained to be determined. MCF-7 and SKBR3 cells were transfected with miR-519d to assess STAT3 mRNA using RT-qPCR and it was found that miR-519d led to an obvious 
A
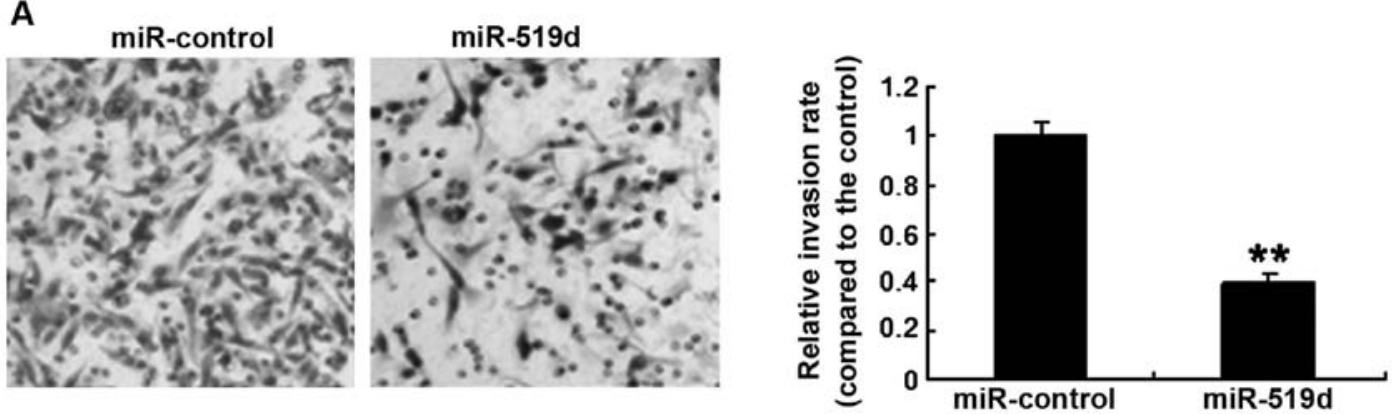

B
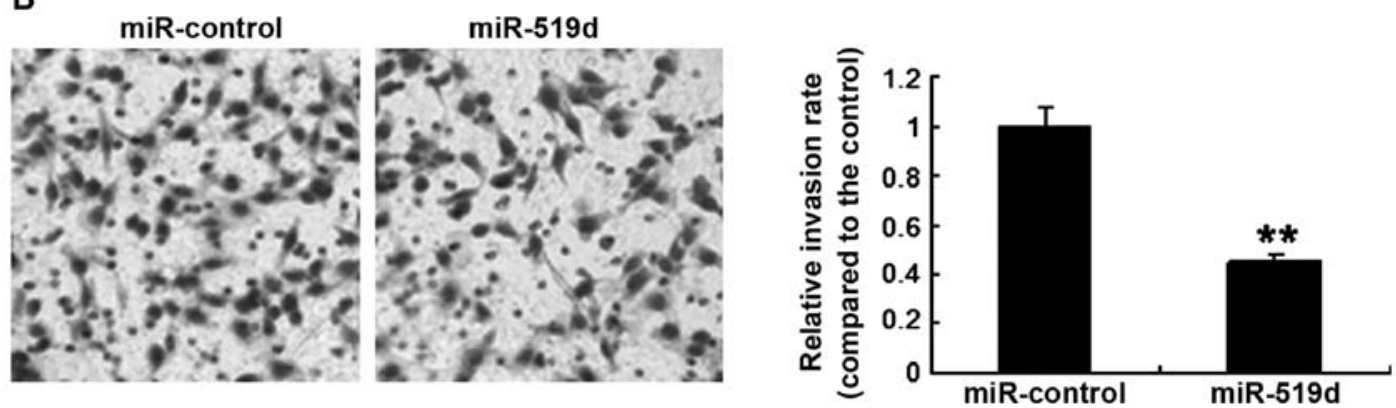

Figure 4. miR-519d inhibits the invasion of breast cancer cells. (A) MCF7 cells were transfected with miR-519d or miR-NC for $48 \mathrm{~h}$ and the cell invasion ability was measured by Matrigel invasion assays. (B) The SKBR3 cells were treated as above and the cell invasion ability was measured by Matrigel invasion assays. Quantification was performed by counting the stained MCF7 and SKBR3 cells that invaded the lower chamber under light microscopy. All the results were reproducible in three independent experiments. ${ }^{* * *} \mathrm{P}<0.01$ as compared to the control.

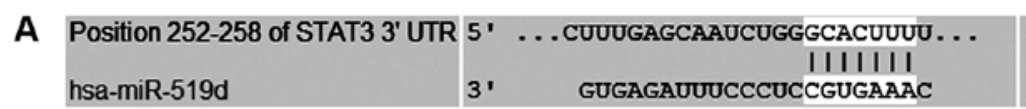

B

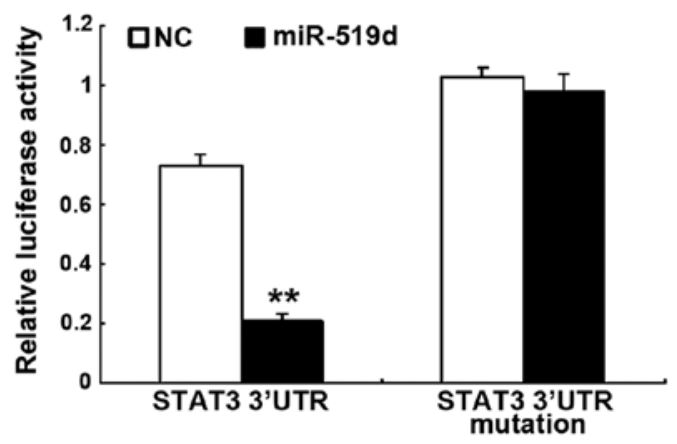

D

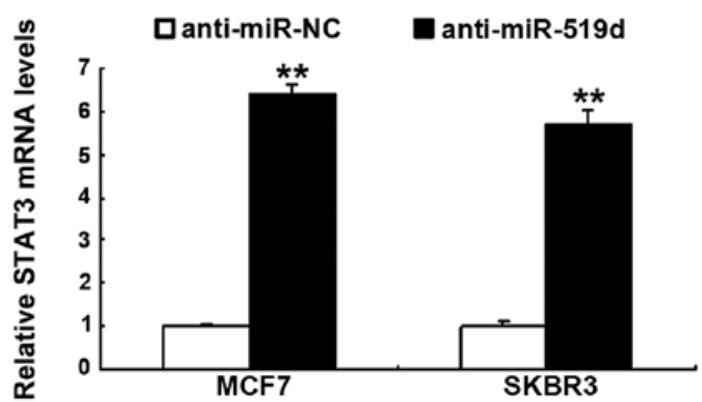

C

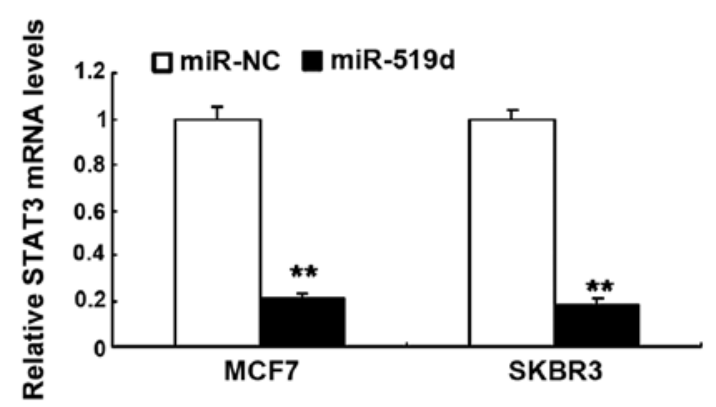

$\mathbf{E}$

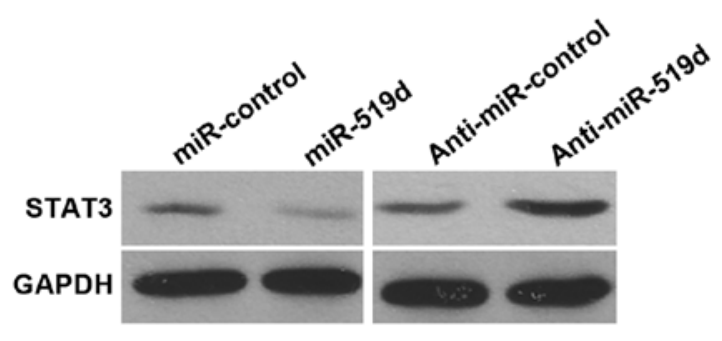

Figure 5. STAT3 is the direct target of miR-519d in breast cancer cells. (A) The miR-519d and miR-519d-binding site in the 3'UTR of STAT3. (B) Luciferase reporter assay with co-transfection of wild-type or mutant 3'UTR and miR-519d or miR-control in MCF7 cells. Firefly luciferase activity of each sample was normalized against Renilla luciferase activity. (C) The effects of miR-519d on the expression of endogenous STAT3 and STAT3 mRNA was assessed by RT-qPCR. (D) The effects of anti-miR-519d on the expression of endogenous STAT3. miR-519d was assessed by RT-qPCR. (E) Western blot analysis was used for monitoring the STAT3 expression in MCF-7 cells $48 \mathrm{~h}$ after the transfection with miR-519d or anti-miR-519d. Data are from three independent experiments and are presented as mean \pm SD. ${ }^{* * *} \mathrm{P}<0.01$. 
A

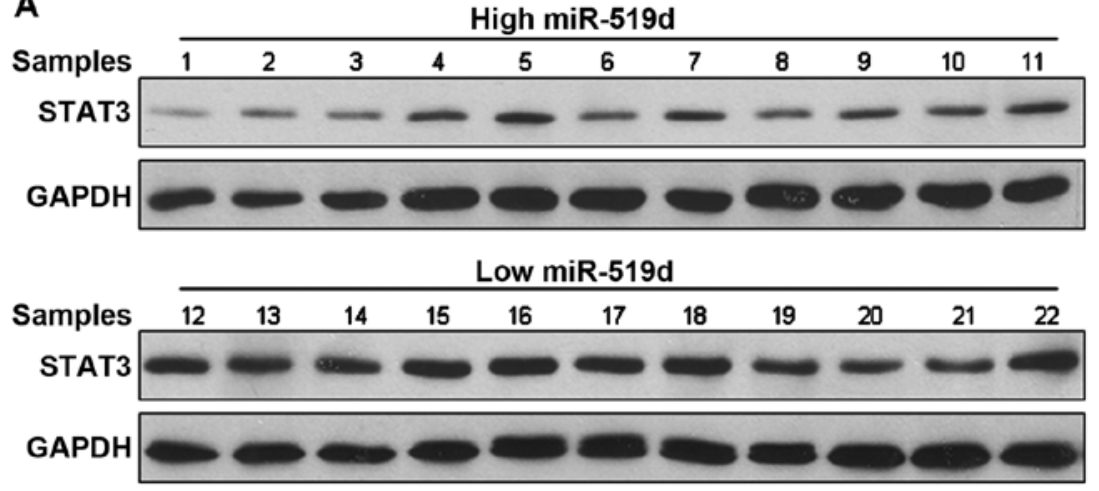

B

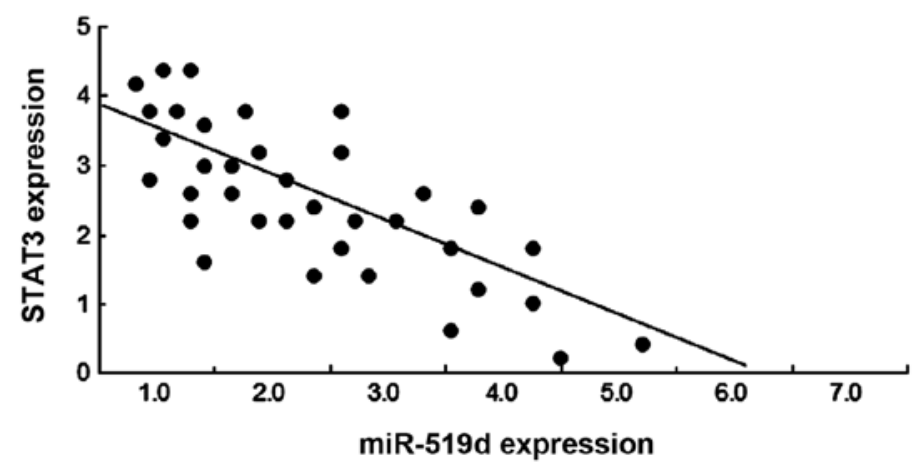

Figure 6. STAT3 protein is negatively correlated with miR-519d in breast cancer tissues. (A) Protein of breast cancer was extracted and performed for STAT3 by western blot analysis. (B) STAT3 protein is negatively correlated with miR-519d in breast cancer tissues $(r=-0.4278 ; \mathrm{P}<0.001)$.

decrease in STAT3 mRNA (Fig. 5C) and the protein (Fig. 5D). By contrast, transfection of anti-miR-519d resulted in an upregulation in STAT3 expression as determined by western blot analysis (Fig. 5E).

miR-519d is negatively correlated to STAT3 in breast cancer tissues. The above data identified that STAT3 is a direct target gene of miR-519d. We then determined whether there is any relationship between STAT3 and miR-519d in breast cancer tissues. Western blot analysis revealed that the breast cancer tissues with a low miR-519d expression had a higher STAT3 protein level while those with a high miR-519d expression had a lower STAT3 protein level (Fig. 6A). As shown in Fig. 6B, there was a significant inverse relationship between STAT3 and miR-519d expression levels in breast cancer tissues.

\section{Discussion}

In recent years, a number of miRNAs that play important roles in breast cancer progression and may be critical diagnostic markers have been identified. One of these is miR-519, which is reported in osteosarcoma (9), ovarian cancer (10) and HCC (11-12). Previous findings have shown that miR-519d is a potential tumor suppressor. However, little is known with regard to the molecular mechanisms involved in the miR-519d modulation in the process of breast cancer. In the present study, we found that miR-519d was frequently downregulated in breast cancer tissues and cell lines. We also found that it suppressed breast cancer growth and invasion, induced apoptosis and cell cycle arrest and its expression was negatively correlated with STAT3 expression in breast cancer.
miR-519d belongs to the chromosome 19 miRNA cluster (C19MC), the largest human miRNA cluster (12). It has been shown that miR-519d plays roles as a negative regulator in tumors. For example, miR-519d in human osteosarcoma is downregulated and then promotes metastasis (10). miR-519d represses ovarian cancer cell proliferation and enhances cisplatin-mediated cytotoxicity in vitro by targeting XIAP (11). miR-519d suppresses cell growth in the hepatocellular carcinoma targeting MKi67 (13). In our study, miR-519d was found to significantly suppress the proliferation of breast cancer cells by induction of apoptosis and cell cycle arrest at $\mathrm{S}$ phase. Furthermore, the growth of xenograft tumors was significantly inhibited after being transfected with miR-519d. These results suggest that miR-519d might act as an inhibitor in the progression of breast tumorigenesis.

STAT3 is regulated by miRNAs in cancer-like miR-130b (18), miR-874 (19), miR-124 (20), miR-200 (20), miR-1181 (21) and miR-7 (22). In the present study, we found STAT3 was verified as a new target gene of miR-519d and its mRNA and protein levels were inhibited in breast cancer cells by miR-519d. Breast cancer tissues were classified as low and high miR-519d expression. The results indicated that expression of miR-519d and STAT3 was negatively correlated.

In conclusion, the results of the present study have demonstrated that the low expression of miR-519d was associated with TNM staging and metastasis of breast cancer. This study also shows that miR-519d plays an important role in the regulation of breast cancer cell proliferation and invasion by downregulating STAT3. Our results indicate that miR-519d is a novel biomarker that can be used to predict the prognosis and progression of breast cancer. 


\section{References}

1. Vislovukh A, Vargas TR, Polesskaya A and Groisman I: Role of 3'-untranslated region translational control in cancer development, diagnostics and treatment. World J Biol Chem 5: 40-57, 2014.

2. van Rooij E and Kauppinen S: Development of microRNA therapeutics is coming of age. EMBO Mol Med 6: 851-864, 2014.

3. Zhu J, Zheng Z, Wang J, Sun J, Wang P, Cheng X, Fu L, Zhang L, Wang $\mathrm{Z}$ and Li Z: Different miRNA expression profiles between human breast cancer tumors and serum. Front Genet 5: 149, 2014.

4. Siegel R, Ma J, Zou Z and Jemal A: Cancer statistics, 2014. CA Cancer J Clin 64: 9-29, 2014.

5. Corcoran C, Friel AM, Duffy MJ, Crown J and O'Driscoll L: Intracellular and extracellular microRNAs in breast cancer. Clin Chem 57: 18-32, 2011.

6. Melo SA and Esteller M: Dysregulation of microRNAs in cancer: Playing with fire. FEBS Lett 585: 2087-2099, 2011.

7. Greene SB, Herschkowitz JI and Rosen JM: Small players with big roles: microRNAs as targets to inhibit breast cancer progression. Curr Drug Targets 11: 1059-1073, 2010.

8. Le Quesne J and Caldas C: Micro-RNAs and breast cancer. Mol Oncol 4: 230-241, 2010

9. Tsai CH, Tsai HC, Huang HN, Hung $\mathrm{CH}$, Hsu CJ, Fong YC, Hsu HC, Huang YL and Tang $\mathrm{CH}$ : Resistin promotes tumor metastasis by down-regulation of miR-519d through the AMPK/p38 signaling pathway in human chondrosarcoma cells. Oncotarget 6: 258-270, 2015.

10. Tsai HC, Su HL, Huang CY, Fong YC, Hsu CJ and Tang CH: CTGF increases matrix metalloproteinases expression and subsequently promotes tumor metastasis in human osteosarcoma through down-regulating miR-519d. Oncotarget 5: 3800-3812, 2014.

11. Pang Y, Mao H, Shen L, Zhao Z, Liu R and Liu P: MiR-519d represses ovarian cancer cell proliferation and enhances cisplatin-mediated cytotoxicity in vitro by targeting XIAP. Onco Targets Ther 7: 587-597, 2014.

12. Fornari F, Milazzo M, Chieco P, Negrini M, Marasco E, Capranico G, Mantovani V, Marinello J, Sabbioni S, Callegari E, et al: In hepatocellular carcinoma miR-519d is up-regulated by p53 and DNA hypomethylation and targets CDKN1A/p21, PTEN AKT3 and TIMP2. J Pathol 227: 275-285, 2012.
13. Hou YY, Cao WW, Li L, Li SP, Liu T, Wan HY, Liu M, Li X and Tang H: MicroRNA-519d targets MKi67 and suppresses cell growth in the hepatocellular carcinoma cell line QGY-7703. Cancer Lett 307: 182-190, 2011.

14. Haura EB, Turkson J and Jove R: Mechanisms of disease: Insights into the emerging role of signal transducers and activators of transcription in cancer. Nat Clin Pract Oncol 2: 315-324, 2005

15. Walker SR, Xiang M and Frank DA: Distinct roles of STAT3 and STAT5 in the pathogenesis and targeted therapy of breast cancer. Mol Cell Endocrinol 382: 616-621, 2014.

16. Clevenger CV: Roles and regulation of stat family transcription factors in human breast cancer. Am J Pathol 165: 1449-1460, 2004.

17. Haghikia A, Hoch M, Stapel B and Hilfiker-Kleiner D: STAT3 regulation of and by microRNAs in development and disease. JAK-STAT 1: 143-150, 2012.

18. Zhao G, Zhang JG, Shi Y, Qin Q, Liu Y, Wang B, Tian K, Deng SC, Li X, Zhu S, et al: Correction: miR-130b is a prognostic marker and inhibits cell proliferation and invasion in pancreatic cancer through targeting STAT3. PLoS One 8: e73803, 2013.

19. Zhang X, Tang J, Zhi X, Xie K, Wang W, Li Z, Zhu Y, Yang L, $\mathrm{Xu} \mathrm{H}$ and $\mathrm{Xu} \mathrm{Z}$ : miR-874 functions as a tumor suppressor by inhibiting angiogenesis through STAT3/VEGF-A pathway in gastric cancer. Oncotarget 6: 1605-1617, 2015.

20. Xiao Y, Wang J, Yan W, Zhou Y, Chen Y, Zhou K, Wen J, Wang Y and Cai W: Dysregulated miR-124 and miR-200 expression contribute to cholangiocyte proliferation in the cholestatic liver by targeting IL-6/STAT3 signalling. J Hepatol 62: 889-896, 2015.

21. Jiang J, Li Z, Yu C, Chen M, Tian S and Sun C: MiR-1181 inhibits stem cell-like phenotypes and suppresses SOX2 and STAT3 in human pancreatic cancer. Cancer Lett 356: 962-970, 2015.

22. Zhang H, Cai K, Wang J, Wang X, Cheng K, Shi F, Jiang L, Zhang Y and Dou J: MiR-7, inhibited indirectly by lincRNA HOTAIR, directly inhibits SETDB1 and reverses the EMT of breast cancer stem cells by downregulating the STAT3 pathway. Stem Cells 32: 2858-2868, 2014. 